\title{
La Description de la nouveauté dans les récits de voyage de Cartier et de Rabelais
}

JEAN-PHILIPPE BEAULIEU

On estime généralement que les récits de voyage du XVIe siècle ont participé de façon importante à la transformation de l'image du monde qui s'est opérée progressivement chez les Européens de la Renaissance. ${ }^{1}$ Lieux de rencontre du connu et de l'inconnu, ces récits cherchaient à transmettre à un lecteur souvent limité par ses systèmes de référence, les impressions et les interrogations des voyageurs relativement aux objets et aux êtres nouveaux rencontrés dans le cadre de cet élargissement du champ "expérientiel" que constituait le voyage d'exploration.

La description de la nouveauté, composante verticale de ces relations, ${ }^{2}$ semble être l'un des éléments les plus susceptibles d'avoir amené des changements sur les plans langagier et épistémologique, en grande partie parce que la pause descriptive est l'endroit du récit où sont énoncées les données linguistiques et référentielles qui révèlent le rapport établi avec le monde nouveau par le rédacteur - que ce dernier soit le voyageur ou son secrétaire. ${ }^{3}$ C'est au niveau de ce rapport "expérientiel" que l'on peut identifier les éléments discursifs qui ont participé à la création d'une nouvelle épistémè au cours des XVIe et XVII' siècles, épistémè "analytico-référentielle," pour reprendre l'appellation de Timothy Reiss, dont les éléments de base consistent en la valorisation de la connaissance empirique de l'univers, au moyen de modèles conceptuels capables de saisir et de formuler les lois naturelles qui sont censées présider à l'organisation du cosmos. ${ }^{4}$

On peut par conséquent supposer que les récits du XVIe siècle qui se présentent au lecteur comme des narrations de voyages effectués dans des régions inconnues offriront des différences notables sur le plan de la description de la nouveauté, selon que les voyages relatés sont réels ou imaginaires. Les récits basés sur des périples historiquement vérifiables posséderaient ainsi des caractéristiques stylistiques que l'on peut rapprocher du discours analytico-référentiel établi définitivement au XVIIe siècle par Francis Bacon. ${ }^{5}$ Les relations imaginaires, quant à elles, moins influencées par les expériences nouvelles, se rattacheraient davantage aux traditions épistémologiques médiévales, où la cosmologie et les modes de 
connaissance s'inscrivent dans un discours conjonctif (ou théocentrique). ${ }^{6}$

L'influence ainsi supposée de l'expérience nouvelle sur le discours descriptif se vérifie lors de l'examen comparatif de la composante descriptive de deux textes presque contemporains, qui ont souvent été rapprochés, dans le passé, pour des raisons de ressemblances thématiques. Il s'agit du BriefRécit de 1545, attribué à Jacques Cartier, et du Quart Livre, de François Rabelais, qui a été publié dans sa version intégrale en $1552 .^{7}$ L'étude stylistique de ces deux textes révèle que les descriptions du Brief Récit sont organisées de façon épistémologique, tandis que celles du Quart Livre le sont de manière rhétorique, c'est-à-dire que les descriptions de Cartier se préoccupent surtout de mettre ou valeur le signifié, c'est-à-dire l'information, tandis que celles de Rabelais accordent une importance particulière au signifiant, c'est-à-dire aux différents procédés de présentation de l'information. On peut en effet constater que les descriptions du Brief Récit s'articulent comme des définitions qui tentent de cerner la nature de l'objet décrit au moyen d'une diversification des éléments informatifs, alors que les descriptions du Quart Livre esquissent une image globale de l'objet décrit en utilisant un minimum de renseignements qu'elles mettent cependant en évidence par des procédés rhétoriques. Les pauses descriptives provenant du récit de Rabelais sont par conséquent plus proches de l'illustration que de la définition.

Les différences d'orientation, que nous venons de situer en termes de définition et d'illustration, trouvent leur prolongement dans d'autres aspects des moments descriptifs et concourent ainsi à mettre en évidence ce qu'on peut considérer comme la principale différence stylistique, soit, d'une part, l'organisation unifiée des descriptions de Rabelais et, de l'autre, la diversité dont font preuve les pauses descriptives provenant du Brief Récit. Cette polarité unité/diversité caractérise les deux séries de descriptions tant sur le plan de l'énoncé que sur celui de l'énonciation.

Sur le plan de l'énoncé, les descriptions de Rabelais se centrent sur un nombre réduit de renseignements, qu'elles exploitent cependant de façon maximale au cours d'une démarche textuelle qui répète et amplifie les renseignements de départ en leur adjoignant des détails synonymiques et en leur faisant subir des métamorphoses de présentation. On peut ainsi reconnaître, dans les descriptions du Quart Livre, une récurrence de "l'identique" toutefois transformée par des procédés rhétoriques qui créent une progression de l'effet. L'énumération est le principal outil stylistique qu'utilise le narrateur afin de présenter les différentès métamorphoses de l'information thématique qu'il a choisie de mettre en relief. Il peut s'agir d'une répétition de sons, de mots ou de phrases syntaxiquement identiques. En conjonction avec d'autres procédés, tels l'intensification des images, l'absence de verbes et l'abondance d'adjectifs souvent hyperboliques, cette succession de propositions synonymiques qu'est l'énumération crée une image thématiquement unifiée, mais peu détaillée, de 
l'objet ou de l'être décrit. La dynamique centralisatrice de cette esquisse descriptive, pléonastique sur le plan de l'énoncé, est de plus renforcée par une énonciation unifiée quant à l'effet, bien que variée en ce qui concerne les moyens utilités pour créer cet effet. Dans l'ensemble des descriptions du Quart Livre, le rédacteur fait effectivement appel de façon continue au registre subjectif pour signaler au lecteur l'ampleur et la nature des réactions émotives que lui et les personnages ont éprouvées devant les réalités nouvelles rencontrées au cours du voyage. Qu'il s'agisse de crainte, dans l'épisode du Physetere, ou d'étonnement, dans la description des habitants

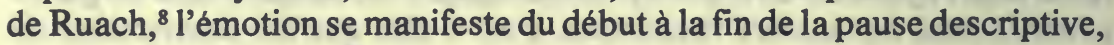
par la présentation et l'accumulation de mots qui possèdent une connotation émotive, ainsi qu'au moyen d'interpellations et de commentaires du narrateur qui indiquent non seulement l'existence d'émotions, mais aussi l'origine et la nature de ces dernières.

Les descriptions de Rabelais sont donc unifiées tant sur le plan de l'énoncé que sur celui de l'énonciation, unité rhétorique qui ne cherche pas à cerner l'essence de la "chose" à décrire, mais illustre certaines caractéristiques de cette dernière, en insistant particulièrement sur leur rapport avec la subjectivité des voyageurs.

Comme nous venons de l'indiquer, il est possible de percevoir distinctement, dans le Quart Livre, l'orientation "monothématique" et unidirectionnelle qu'adopte la démarche descriptive du conteur. Il s'agit d'une dynamique paradigmatique, où l'élan descriptif est suscité par une continuelle permutation du "semblable." Les descriptions de Cartier, par contre, se situent sur le plan syntagmatique du langage où le "différent" succède au "différent," tant sur le plan de l'énoncé que sur celui de l'énonciation, pour offrir une image composite de l'objet décrit. Au lieu de se superposer, comme dans le Quart Livre, pour arriver à un patron idéel de la "chose" dont on parle, les informations fournies par le relateur se juxtaposent les unes aux autres de façon linéaire à l'intérieur d'un mouvement du regard descriptif qui renseigne le lecteur sur plusieurs aspects de la nouvelle réalité rencontrée.

On peut ainsi reconnaître, dans le Brief Récit, une diversification de l'information qui projette dans l'espace textuel une image détaillée et plutôt complexe de l'objet décrit. Ce dernier n'est pas représenté au moyen d'une esquisse linguistique pléonastique, mais plutôt à l'aide de divers procédés stylistiques qui créent une image nettement différenciée de la nouvelle réalité. Dans le moment descriptif portant sur l'adhothuys, ${ }^{9}$ par exemple, on retrouve des renseignements sur les dimensions, la couleur, le lieu d'habitation, la répartition géographique et même le goût de cet animal marin, présentés au moyen de comparaisons, d'évaluations perceptuelles et de compte-rendus. Cet inventaire rapide signale bien la variété du matériel linguistique inscrit dans un espace textuel limité. 
Une telle constatation révèle l'effort que fait le "relateur" pour transmettre au lecteur un ensemble varié de signifiés qui correspondrait à l'image cognitive que les voyageurs se sont faits de la réalité nouvelle. Quoique l'on puisse sentir ici une volonté de définir, donc de cerner l'essence de ce qui est décrit, le texte, en multipliant les détails et les procédés stylistiques, donne naissance, non pas à une image précise et intégrée de l'objet, mais à une création linguistique hétérogène, qui présente une "chose" hybride dont l'essence et l'existence prennent appui, du moins dans le texte, sur une juxtaposition de caractéristiques appartenant à d'autres objets de l'univers. Dans la description de l'adhothuys, par exemple, l'animal que le texte évoque semble ainsi être la résultante d'une combinaison de traits physiques appartenant à d'autres éléments de l'univers que connaît le "relateur." Ce dernier nous présente en effet un poisson sans nageoires, qui ressemble à un cétacé et à un chien, blanc comme neige et qui vit entre deux eaux. La juxtaposition de ces attributs nous fait voir l'animal plus par ses contours analogiques, c'est-à-dire par les correspondances avec l'univers que l'humain croit retrouver dans la bête, que par ses caractéristiques propres.

À cette diversité de l'énoncé correspond une diversification des registres de l'énonciation qui rend compte du sentiment d'ambiguïté entourant le statut des objets nouveaux dont parle le "relateur." Ce dernier présente certaines informations avec objectivité, tandis que pour d'autres, le ton qu'il utilise devient lyrique, admiratif ou méprisant. La présence d'un tel mouvement de va-et-vient entre le registre objectif et le registre émotif souligne la nature changeante de l'attitude du "relateur" face aux réalités nouvelles.

La polarité unité/diversité, qui caractérise, d'une part, les descriptions du Quart Livre et, de l'autre, celles provenant du Brief Récit, peut s'exprimer dans les termes d'une autre opposition qui tient compte de façon plus globale de la dynamique interne des moments descriptifs. Il s'agit de la dualité centrifuge/centripète, modèle conceptuel binaire qui nous semble intéressant parce qu'il indique que l'ensemble des éléments qui forment les descriptions de Cartier possèdent une tendance à l'expansion, tandis que ceux qui composent celles de Rabelais font preuve d'une énergie centralisatrice. Cela signifie que même si certaines caractéristiques des deux séries de descriptions se ressemblent, leur façon de s'organiser les oppose selon une importante différence d'orientation.

Une telle polarité peut s'expliquer par la correspondance du choix organisationnel des éléments stylistiques et d'un mode de connaissance de l'univers. Dans la perspective où le texte représente un microcosme dans lequel les deux écrivains transcrivent les composantes du macrocosme (l'univers) au moyen d'un réseau de correspondances analogiques, ${ }^{10}$ on peut considérer la transcription du "grand" au "petit" comme une opéra- 
tion de médiatisation qui met en évidence la vision du macrocosme que possède le rédacteur. Il est par conséquent possible de rapprocher les caractéristiques stylistiques d'une description - cette dernière étant la transcription linguistique d'une "chose" appartenant au macrocosme - et la conception que se font les deux écrivains du langage comme outil de transposition. Une telle inscription des données descriptives dans un discours épistémologique global permet d'expliquer les différences qui caractérisent les deux récits, en identifiant l'épistémè à laquelle appartient le langage de chacun des rédacteurs. ${ }^{11}$

Comme nous l'avons déjà noté, les descriptions du Quart Livre font preuve d'une grande force de cohésion interne qui se traduit par une présentation pléonastique des éléments linguistiques. L'impression d'ordonnance et de stabilité qui résulte de cette organisation textuelle suggère que Rabelais propose au lecteur une transcription linguistique univoque et fidèle du monde qu'il décrit. Ce procédé de transposition, dans lequel l'ordonnance centripète des éléments stylistiques semble reproduire une ordonnance similaire du macrocosme, nous indique que le rédacteur du Quart Livre reconnaît, d'une part, le pouvoir de représentation du langage par rapport à son univers imaginaire et, de l'autre, la possibilité de réduire la correspondance macrocosme/microcosme à un principe premier vers lequel tendent toutes les composantes de ces deux systèmes analogiques.

La place et l'importance de l'attribution de noms dans les pauses descriptives du Quart Livre indiquent en effet que, pour le rédacteur, le mot possède le pouvoir d'évoquer directement une réalité précise: à chaque "chose" - même nouvelle sur le plan de l'imaginaire - correspond un mot capable d'englober la nature et la fonction de cette "chose." Rabelais admet donc que le langage est à même de transcrire le macrocosme en lui imposant toutefois, au cours du processus de transcription, l'encadrement d'une logique rhétorique plus conceptuelle "qu'expérientielle," qui est d'ailleurs censée refléter les lois et l'organisation de l'univers, où tout concourt à la reconnaissance de la présence première et ultime de Dieu.

Dans une telle perspective, la description de la nouveauté ne présente aucune difficulté particulière pour le conteur. Même s'ils sont périphériques par rapport à l'univers connu, les mondes nouveaux explorés par les voyageurs du Quart Livre sont soumis aux mêmes lois universelles d'ordre divin et, par conséquent, au même système analogique qui permet d'assimiler linguistiquement le "différent" au "semblable" en l'inscrivant dans une organisation hiérarchique pyramidale dont Dieu est le sommet et la condition d'existence. L'économie centripète des descriptions de Rabelais reflète donc une dynamique épistémologique conjonctive dont les éléments tout comme ceux du texte - tendent vers un point commun en reproduisant l'organisation théocentrique de l'univers. 
Comme cette conception du langage et du cosmos relève d'un principe unificateur qui trouve son origine dans le symbolisme universel énoncé par saint Augustin, ${ }^{12}$ nous pouvons donc conclure que les descriptions de Rabelais participent de l'épistémè médiévale.

Les descriptions du Brief Récit, pour leur part, se caractérisent par la variété des moyens stylistiques mis en oeuvre pour décrire la nouvelle réalité. La transcription de cette dernière dans le microcosme du texte s'avère ainsi une opération linguistique qui se distingue plus par l'expansion de ses éléments constitutifs que par leur concentration. Il s'agit d'une stratégie textuelle qui met en évidence la difficulté qu'éprouve le "relateur" à décrire brièvement la nouveauté au moyen de procédés stylistiques univoques. Cartier reconnait en effet le caractère approximatif de son langage descriptif, en ne cessant d'ajouter des renseignements à son texte, de façon parfois anarchique, afin d'appréhender une réalité qui semble continuellement se dérober à son effort langagier.

Le Nouveau-Monde ne semble guère vouloir s'assimiler à l'Ancien sur le plan analogique, d'où la nécessité de singulariser les choses à l'aide de procédés épistémologiques qui constituent en fin de compte un aveu partiel de l'impossibilité du langage à saisir directement la réalité. Bien que l'ordre universel de similitude et de hiérarchie des choses semble ainsi rompu de façon irrémédiable, le "relateur" réussit néanmoins à situer le "différent" en l'incorporant à un discours descriptif dont les prémisses, qui s'inscrivent dans la tradition analogique médiévale, mènent à des constructions scripturaires qui tiennent de plus en plus compte de l'individualité des choses, saisie par l'expérience immédiate. De ce fait, Cartier fait preuve d'une orientation intellectuelle "renaissante," car tout en étant conscient de l'arbitraire relatif du langage, le "relateur" tente de cerner la nature des choses avec le principal outil de communication qu'il connait. Cette attitude visà-vis de la réalité du Nouveau-Monde, qui donne naissance à un discours descriptif diversifié, se rapproche ainsi "de l'ouverture à la variété des choses" que Jérôme Cardan identifiait comme caractérisant la révolution intellectuelle que les Européens du XVIe siècle ont eu conscience de vivre. ${ }^{13}$

À partir des informations présentées précédemment, il apparaît donc fort probable que les contraintes "expérientielles" du voyage ont influencé le rédacteur du Brief Récit en lui imposant la problématique de la description de la nouveauté, problématique que Rabelais, en décrivant son univers imaginaire, n'a pas rencontrée, et dont la résolution textuelle se présente chez Cartier comme une des étapes importantes menant à la formation d'une nouvelle épistémè. 


\section{Notes}

1 Il faut insister ici sur le mot "progressivement," qui souligne l'étendue temporelle d'un processus épistémologique dont les effets culturels ne se sont manifestés que peu à peu. À ce sujet, voir Lucien Febvre, Le Problème de l'incroyance au XVIe siècle (Paris: Albin-Michel, 1942), p. 422-423; John H. Elliott, "Renaissance Europe and America: a Blunted Impact?" in First Images of America, éd. Fredi Chiappelli (Berkeley: University of California Press, 1976), I, p. 17.

2 Gérard Genette, "Frontières du récit," in Figures II (Paris: Seuil, 1969), p. 56.

3 La problématique de l'influence de l'expérience sur la description de la nouveauté est énoncée par Alexandre Cioranescu, "La Découverte de l'Amérique et l'art de la description," Revue des Sciences Humaines, 106 (1962), p. 161.

4 Timothy Reiss, The Discourse of Modernism (Ithaca: Cornell University Press, 1982), p. 31.

5 Pour un exposé des éléments qui constituent l'empirisme de Bacon, voir Reiss, chapitre 6, "The Masculine Birth of Time," p. 198-224.

6 Reiss, p. 72; Peter Haidu, "Repetition: Modern Reflections on Medieval Aesthetics," Modern Language Notes, 92 (1977), p. 878-879.

7 Brief Récit in The Voyages of Jacques Cartier, ed. H. P. Biggar (Ottawa: Publications of the Public Archives of Canada, 1924); François Rabelais, Le Quart Livre, ed. R. Marichal (Genève: Droz, 1947). Nous utiliserons le nom de Cartier comme un "vocable" qui désigne l'auteur du Brief Récit. Au sujet de la paternité de ce texte, voir André Berthiaume, La Découverte ambiguë (Montreal: Pierre Tisseyre, 1976), p. 40.

8 Rabelais, p. 152 et $182-183$.

9 Cartier, p. 117.

10 Guy Demerson, “Rabelais et l'analogique," Études Rabelaisiennes, 14 (1977), p. 25.

11 Reiss, p. 32.

12 Johan Chydenius, "La Théorie du symbolisme universel," Poétique, 23 (1975), p. 325; Alfonso Maierù, "'Signum' dans la culture médiévale," in Sprache und Erkenntnis im Mittelalter, éd. J. Beckman et L. Honnefelder (Berlin: De Gruyter, 1981), I, p. 57.

13 Cité par Michel Mercier, Le Roman féminin (Paris: Presses universitaires de France, 1976), p.34. 